\title{
Position of dorsal root ganglia in the lumbosacral region in patients with radiculopathy
}

\author{
Hyun Seog Moon ${ }^{2}$, Yeon Dong Kim², Bang Hoon Song ${ }^{1}$, Young Deog Cha ${ }^{1}$, Jang Ho Song ${ }^{1}$, and \\ Mi Hyeon Lee ${ }^{1}$ \\ ${ }^{1}$ Department of Anesthesiology and Pain Medicine, Inha University Hospital, Incheon, ${ }^{2}$ MHS Department of Pain Medicine, \\ Gwangju, Korea
}

Background: When applying pulsed radiofrequency on dorsal root ganglia for treating chronic lower back pain, maximum efficiency can be expected when a needle is placed $1-2 \mathrm{~cm}$ peripheral to the dorsal root ganglion. The object of this study is to analyze images taken after adding contrast to transforaminal epidural injection, categorize root ganglia according to anatomical position, and provide a reference for efficient needle positioning in applying pulsed radiofrequency on dorsal root ganglia.

Methods: From January 2008 to January 2009, 457 patients who visited our hospital for root pain or radiculopathy were treated with transforaminal epidural injection on the nerve roots based on the dermatome of the painful area. Anteroposterior views were taken after injection of contrast. A virtual line was made by connecting the internal and external parts of the spinal pedicle from the contrast images. Then the dorsal root ganglia were categorized as intraspinal (IS), intraforaminal (IF), or extraforaminal (EF).

Results: In the fourth lumbar spine, dorsal root ganglia positions were $48 \%$ IF, $41 \%$ IS, and $6 \% \mathrm{EF}$. In the fifth lumbar spine, dorsal root ganglia positions were $75 \%$ IF, $10 \%$ IS, and $6 \%$ EF. In the first sacral spine, dorsal root ganglia locations were $8 \%$ IF and $83 \%$ IS.

Conclusions: Positional categorization of dorsal root ganglia according to contrast images was proven to be good anatomical references for effective radiofrequency or blocking of dorsal root ganglia. (Korean J Anesthesiol 2010; 59: 398-402)

Key Words: Catheter ablation, Dorsal root ganglion, Epidural injection.

\footnotetext{
Received: June 8, 2010. Revised: 1st, June 28, 2010; 2nd, July 26, 2010. Accepted: August 3, 2010.

Corresponding author: Mi Hyeon Lee, M.D., Department of Anesthesiology and Pain Medicine, Inha University Hospital, Sinheung-dong 3-ga, Jung-gu, Incheon 400-711, Korea. Tel: 82-32-890-3968, Fax: 82-32-881-2476, E-mail: md1212@naver.com

This work was supported by Inha University.

(c) This is an open-access article distributed under the terms of the Creative Commons Attribution Non-Commercial License (http:// creativecommons.org/licenses/by-nc/3.0/), which permits unrestricted non-commercial use, distribution, and reproduction in any medium, provided the original work is properly cited.
} 


\section{Introduction}

Dorsal root ganglia are sensitive to ischemia caused by physical compression or chemical stimulation induced by inflammation and a critical condition that can cause chronic pain in lower extremity [1].

Dorsal root gangliocytes are more sensitive to heat than other nervous tissues. It is known that pulsed radiofrequency can selectively block sensory nerves while minimizing the destruction of motor nerves. Sluijter [2] reported that the placement of a cannula $1-2 \mathrm{~cm}$ peripheral to the dorsal root ganglia could result in maximum effect when pulsed radiofrequency was applied on dorsal root ganglia of the spinal cord.

Kikuchi et al. [1] classified anatomical positions and variations of dorsal root ganglia into intraspinal (IS), intraforaminal (IF), and extraforaminal (EF). No studies, however, have yet classified the frequency of dorsal root ganglia in each position at the lumbosacral region. As such, in this study, we included a higher number of patients with radiculopathy than in previous studies, injected contrast into those patients during transforaminal epidural block, analyzed the patterns of contrast, and classified anatomical positions of dorsal root ganglia into left and right. This classification helps in determining the best location for inserting a cannula during pulsed radiofrequency.

\section{Materials and Methods}

After obtaining approval from the institutional ethics
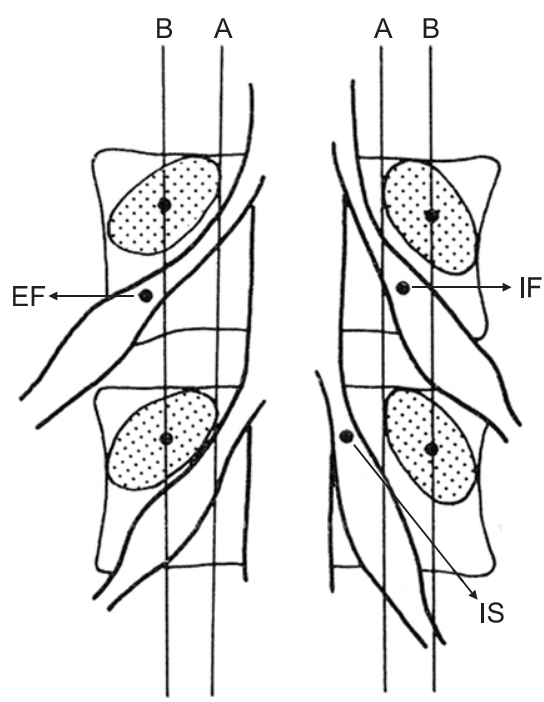

Fig. 1. Positions of dorsal root ganglia (DRG) were determined by two schematic lines and classified into three types. Line A: aligning the medial borders of L4 and L5 pedicles, Line B: aligning the centers of L4 and L5 pedicles, Intraspinal type (IS) : DRG located proximal to line A, Intraforaminal type: DRG located between line A and B, Extraforaminal type: DRG located distal to line B. committee, we studied 457 patients with pain in the lower extremity due to radiculopathy among the patients who visited this author's hospital from January 2008 to January 2009. Participants consist of 190 male and 267 female patients. The average age of males was 55.6, and average age of females was 58.4 .

Patients with hemostatic disorder, infection, allergy to medication, patients who were pregnant, patients that had contraindications to steroids, and patients who refused to provide written agreement were excluded from the study. Transforaminal epidural block was performed based on the dermatomal region of the nerve root of the experienced pain, contrast was injected on the area, and pre- and post-block images were obtained.

With the patients in a prone position, the relevant area of lesion was sufficiently disinfected, and sterilized drape was placed to cover the area. The skin was anesthetized with $6 \mathrm{ml}$ of $1 \%$ lidocaine, then a 21 -gauge, $10 \mathrm{~cm}$ needle was inserted in the superior articular end of the spinal body until it reached the superior articular process. The needle was slid into the foramen, then after checking using physiological saline that there was no resistance, contrast was injected. Cases were classified as contrast failure when severe stimulating pain or resistance occurred, subdural space was filled with contrast, and intravasucular injection of contrast was suspected.

On the contrast image, an imaginary line linking the interior and exterior side of the superior and inferior spinal body was drawn, and the position of the dorsal root ganglia was classified into IS, IF, and EF based on the starting position of the dorsal root ganglia (Fig. 1 and 2) [1,3].

Data was analyzed using SPSS (version 14.0, SPSS Inc., Chicago, IL, USA), and all values were expressed in mean \pm SD or in the number of patients. Demographic comparison was performed using one ANOVA with a significance level of $\mathrm{P}<0.05$.

\section{Results}

There was no significant difference in age, sex, height, and body weight among patients from whom contrast images of the fourth and fifth lumbar and the first sacral region was obtained (Table 1).

On the fourth lumbar region, IS, IF, and EF were $34.2 \%, 55.3 \%$, and $7.9 \%$, respectively on the right side and $54.2 \%, 37.5 \%$, and $4.2 \%$, respectively on the left side. It was difficult to classify $2.6 \%$ of dorsal root ganglia on the left side and $4.2 \%$ on the right side. On the fifth lumbar region, IS, IF, and EF were $10.6 \%, 75.4 \%$, and $6.5 \%$, respectively on the right side and $10.8 \%, 75.7 \%$, and $6.1 \%$, respectively on the left. It was difficult to categorize $7.5 \%$ of dorsal root ganglia on the left side and $7.4 \%$ on the right. On the first sacral vertebra, IS and IF were $84.6 \%$ and $3.8 \%$, respectively 

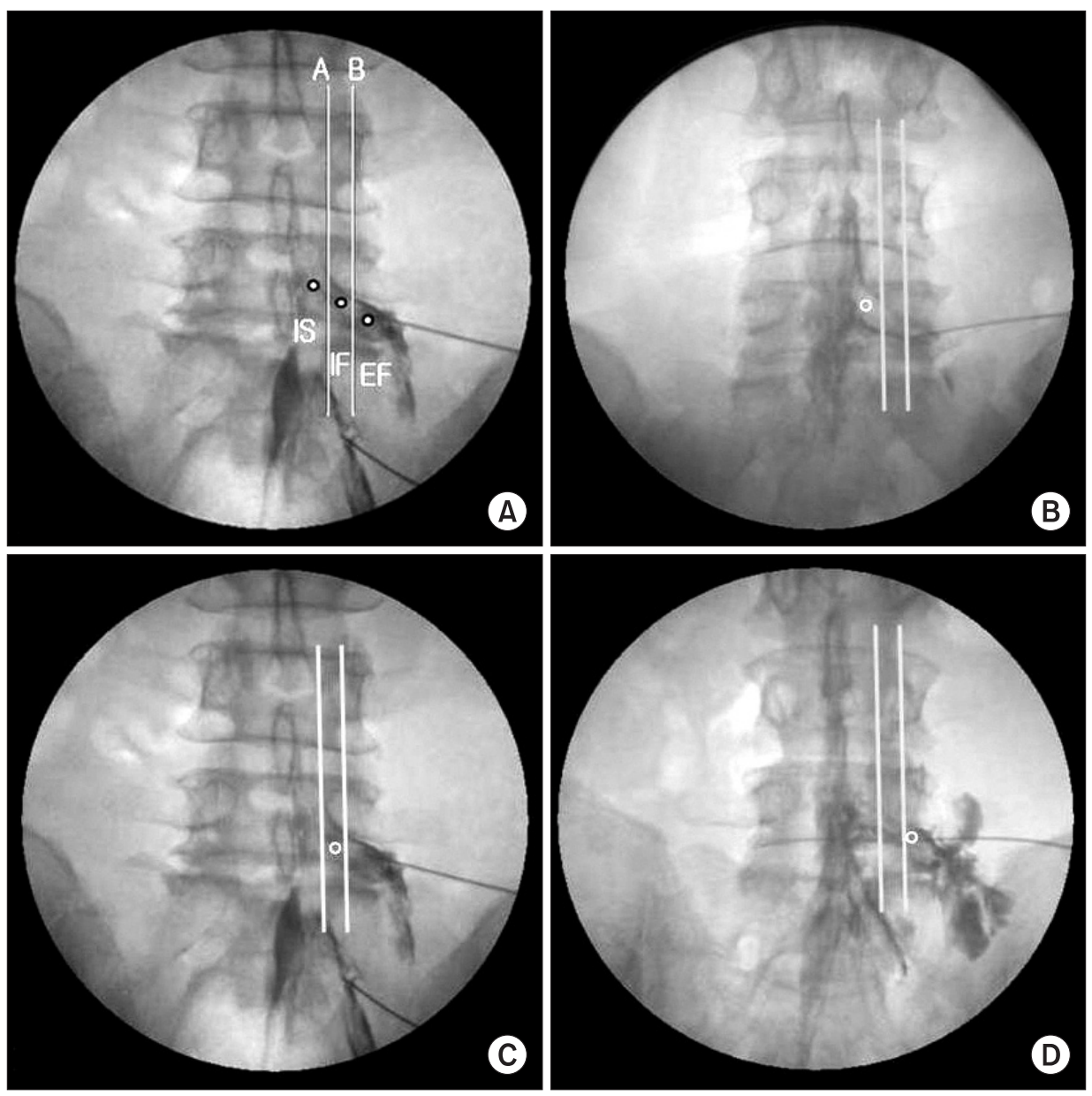

Fig. 2. Epidurogram showing the three types of dorsal root ganglia (DRG) with contrast injection in anteroposterior view. (A) three types of DRG, (B) intraspinal type, (C) intraforaminal type, (D) extraforaminal type.

Table 1. Demographic Data of Study Patients

\begin{tabular}{lccc}
\hline & L4 $(\mathrm{n}=62)$ & L5 $(\mathrm{n}=347)$ & $\mathrm{S} 1(\mathrm{n}=48)$ \\
\hline Age $(\mathrm{yr})$ & $54.9 \pm 10.2$ & $59.8 \pm 12.3$ & $56.5 \pm 11.2$ \\
Sex $(\mathrm{M} / \mathrm{F})$ & $27 / 35$ & $141 / 206$ & $22 / 26$ \\
Height $(\mathrm{cm})$ & $161.9 \pm 8.2$ & $158.1 \pm 7.6$ & $162.0 \pm 5.8$ \\
Weight $(\mathrm{kg})$ & $65.3 \pm 10.5$ & $66.4 \pm 10.1$ & $62.5 \pm 5.8$ \\
\hline
\end{tabular}

Values are mean \pm SD or number of patients. M: male, F: female.

in the right side. As for the left side, IS and IF were $81.8 \%$ and $13.6 \%$, respectively. $11.5 \%$ of dorsal root ganglia on the left side and $4.5 \%$ on the right side were difficult to classify (Table 2).

\section{Discussion}

Back pain is one of the most common chronic pains [4]. The major cause of chronic back pain is secondary inflammation induced by stimulation of the nerve root and dorsal root ganglia caused by lumbar disc herniation.

Embryologically, dorsal root ganglia originates in the neural crest during its embryotic phase and moves toward the peripheral region as it grows [5]. Normally, it is located in the interior of the foramen exterior immediately under the vertebral pedicle. In the sacral nerve, however, it exists in the spinal canal [6]. Unlike dorsal root ganglia in the inferior lumbar region, it is located in the dorsal area of the foramen in the superior lumbar area.

Ventral root afferent that causes pain in the ventral root of the spinal nerve comes from dorsal root ganglia, forms a loop, goes into the ventral root, and then goes back to the spinal cord. Thus, the reason why pain was not relieved even after posterior rhizotomy was ascertained [7], and the pain could be mitigated by excision of the dorsal root ganglia.

It is known that since dorsal root gangliocytes are more sensitive to heat than other nervous tissues, thermal coagulation through high-frequency stimulation could relatively well preserve motor nerves as well as intrinsic senses and tactile sensation, thereby reducing deafferentation pain [8]. Despite this, the procedure may cause adverse effects such as reduced sensory and neuritis-like symptoms for certain periods after the procedure because it is a surgical procedure that can interrupt certain nerves. Moreover, it may also result in paresthesia, hyperpathia, and allodynia because of aggravation of pains 
Table 2. Incidence and Laterality of Types of the L4, L5 and S1 Dorsal Root Ganglion

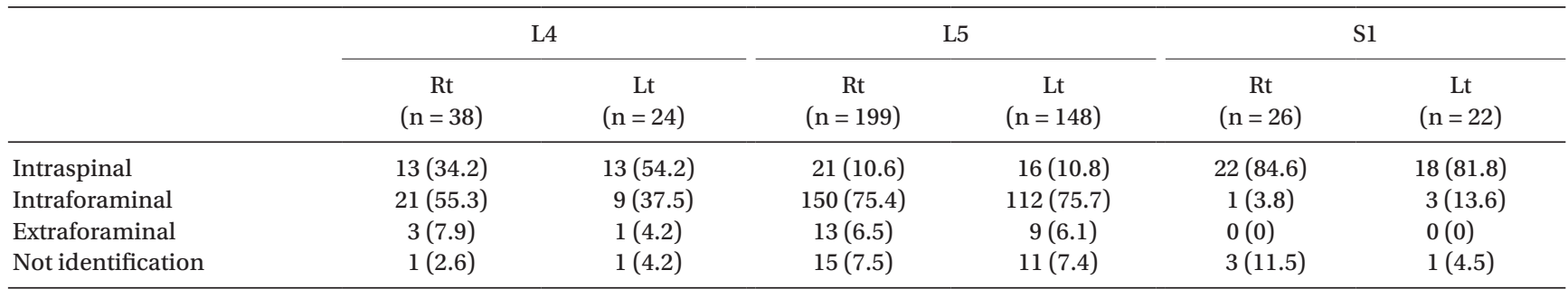

Values are number of patients (\%).

during the neuranagenesis.

In 1998, Sluijter [9] developed pulsed radiofrequency. In pulsed radiofrequency, the maximum temperature of the electrode tip of nerve blocking needle is limited to $42^{\circ} \mathrm{C}$, thereby preventing heat damage to the tissue, and high voltage is used as well to maximize the electromagnetic field $[10,11]$. Furthermore, pulsed radiofrequency causes less pain and destruction of the nerve during the procedure and is more reversible and less destructive than high-frequency heat coagulation. Moreover, it can be applied to risky areas [12]. Sluijter [2] reported that best results could be achieved when the cannula was placed $1-2 \mathrm{~cm}$ peripheral to the dorsal root ganglia and pulsed radiofrequency was performed on the dorsal root ganglia. Thus, it is clinically important to study the anatomical difference of dorsal root ganglia to determine the best location in which the cannula should be inserted for pulsed radiofrequency.

Dorsal root ganglia can be classified according to the area where it starts. It can therefore be categorized into IS, where half or more of the ganglion exists in the spinal canal, IF, where the ganglion exists in the foramen, and EF, where most of the ganglion exists outside the foramen $[1,3]$.

In this study, dorsal root ganglia were classified at the level of sacral spine and their frequency was analyzed. In the fourth lumbar area, IF was most common in the right side, existing in $55.3 \%$ of the subjects whereas in the left side, IS was $54.2 \%$. In the fifth lumbar area, IF was the most common both in right and left side, existing in $75.4 \%$ and $75.7 \%$ of the subjects, respectively. For the first sacra, both in the right and left side, IS was most common, $84.6 \%$ and $81.8 \%$, respectively. Overall, in the fourth and fifth lumbar area, the proportion of dorsal root ganglion was in descending order of IF, IS, and EF. In the first sacra, it was in descending order of IS and IF, and EF was not found. Kikuchi et al. [1] analyzed the type and frequency of dorsal root ganglion using cadavers and reported that in the fourth and fifth lumbar region, most of the dorsal root ganglion was IF ( $86.1 \%$ and $72.8 \%$, respectively), and for the first sacra, most of it was IS (77.3\%). Thus, the result of this study of patients with radiculopathy is consistent with the results of previous studies that suggested that dorsal root ganglia were located more proximal to the sacral vertebra than to lumbar $[1,3]$. Also, since blood vessels are abundant in the sacral canal of the sacral vertebra, intravascular injection of contrast is particularly frequent [2]. Because of this, it is believed that contrast is more common in sacral vertebra than in lumbar vertebra.

Several recent studies reported that dorsal root ganglia in the proximal area were related to radiculopathy $[5,13]$. Although the mechanism of sciatica is diverse, the spatial relationship between a nerve and its neighboring tissues is basically important. Dorsal root ganglia of the IS type is most susceptible to mechanical and chemical stimulation because nerve root canal of the IS type, particularly in the subarticular region, is narrowest and consequently stable, and motional compression can be more intense [3]. In this study, the proportion of proximal dorsal root ganglia in the fourth lumbar vertebra in the left side was higher than in other previous studies. It is believed that the proportion of proximal dorsal root ganglia was higher than in previous anatomical studies because such studies done in the past were performed on randomly selected patients but this study was conducted on patients who complained of symptoms. However, the exact causes of dorsal root ganglia are difficult to ascertain. More studies using imaging as well as attempts for anatomical classification are required.

In conclusion, the positional classification of dorsal root ganglia obtained by injecting contrast during transforaminal epidural block would be useful as anatomical or imaging reference data for treatment using high-frequency stimulation for the treatment of dorsal root ganglia and for transforaminal epidural block. Moreover, the classification of incidence rate according to the type of dorsal root ganglia in the sacral vertebra would be helpful for understanding radiculopathy.

\section{References}

1. Kikuchi S, Sato K, Konno S, Hasue M. Anatomic and radiographic study of dorsal root ganglia. Spine (Phila Pa 1976) 1994; 19: 6-11.

2. Sluijter ME. Radiofrequency, part I: the lumbosacral region. Meggen, Flivopress SA. 2001, pp 119-38.

3. Hasue M, Kunogi J, Konno S, Kikuchi S. Classification by position of dorsal root ganglia in the lumbosacral region. Spine (Phila Pa 1976) 1989; 14: 1261-4.

4. Abdi S, Datta S, Trescot AM, Schultz DM, Adlaka R, Atluri SL, et 
al. Epidural steroids in the management of chronic spinal pain: a systematic review. Pain Physician 2007; 10: 185-212.

5. Vanderlinden RG. Subarticular entrapment of the dorsal root ganglion as a cause of sciatic pain. Spine (Phila Pa 1976) 1984; 9: 1922.

6. Williams PL, Warwick R. Gray's anatomy. 36th ed. London, Churchill Livingstone. 1980, p 1086.

7. Hosobuchi Y. The majority of unmyelinated afferent axons in human ventral roots probably conduct pain. Pain 1980; 8: 167-80.

8. Kline MT. Stereotactic radiofrequency lesions as part of the management of pain. Orlando, Deutsch Press. 1992, pp 29-32.

9. Sluijter ME. Pulsed radiofrequency. Anesthesiology 2005; 103: 1313; author reply 1313-4.
10. Malik K, Benzon HT. Radiofrequency applications to dorsal root ganglia: a literature review. Anesthesiology 2008; 109: 527-42.

11. Hamann W, Abou-Sherif S, Thompson S, Hall S. Pulsed radiofrequency applied to dorsal root ganglia causes a selective increase in ATF3 in small neurons. Eur J Pain 2006; 10: 171-6.

12. Sluijter ME, Cosman ER, Rittman WB, van Kleef M. The effects of pulsed radiofrequency fields applied to the dorsal root ganglion: a preliminary report. Pain Clin 1998; 11: 109-17.

13. Jeon YT, Hwang JW, Lim YJ, Kim YC, Lee SC. Epidural hyaluronic acid in a rat model of chronic dorsal root ganglion compression. Korean J Anesthesiol 2004; 46: 462-6. 